\title{
Controlling Intramolecular Conformation through Nonbonding Interaction for Soft-Conjugated Materials: Molecular Design and Optoelectronic Properties
}

Yuanfang Cheng ${ }^{1}$, Yuanyuan ${ }^{1}{ }^{1}$, Yuting Tang ${ }^{l}$, Chao Zheng ${ }^{l}$, Yifang Wan ${ }^{1}$, Wei Huang ${ }^{2}$, Runfeng Chen $^{1 *}$

${ }^{1}$ Key Laboratory for Organic Electronics and Information Displays \& Institute of Advanced Materials (IAM), Jiangsu National Synergetic Innovation Center for Advanced Materials (SICAM), Nanjing University of Posts and Telecommunications (NUPT), Nanjing, 210023, P.R. China.

${ }^{2}$ Key Laboratory of Flexible Electronics (KLOFE) \& Institute of Advanced Materials (IAM), Jiangsu National Synergetic Innovation Center for Advanced Materials (SICAM), Nanjing Tech University (Nanjing Tech), Nanjing 211816, P.R. China. 


\section{Theoretical and Computational Methodology}

Computational methods. All the density functional theory (DFT) calculations were performed on Gaussian 09 revision D.01 package. The molecular structures were fully optimized by the Becke's three-parameter exchange functional along with the Lee-Yang-Parr correlation functional (B3LYP) for neutral, cationic, and anionic states molecules at the standard split valence plus polarization function $6-31 \mathrm{G}(\mathrm{d})$ basis set. Molecular structures at the lowest singlet excited states $\left(\mathrm{S}_{1}\right)$ were optimized via time-dependent DFT (TD-DFT) of TD-B3LYP/6-31G(d). ${ }^{1}$ Vibrational frequencies analysis was performed for the optimized geometries to confirm all stationary points are minima (zero imaginary frequencies) on the respective potential energy hypersurfaces. The frontier molecular orbital energy levels and isodensity surface of the highest occupied molecular orbital (HOMO) and the lowest unoccupied molecular orbital (LUMO) were calculated on the optimized structures at the ground state $\left(\mathrm{S}_{0}\right)$. The band-gap $\left(E_{\mathrm{g}}\right)$ was achieved from the energy difference between HOMO and LUMO of the compound. The photophysical properties including absorption and emission spectra were calculated using TD-B3LYP/6-31G(d) at the optimized ground $\left(\mathrm{S}_{0}\right)$ and excited $\left(\mathrm{S}_{1}\right)$ states, respectively. Absorption and emission spectra in vacuum were simulated by Gaussian functions with a half-width of $3000 \mathrm{~cm}^{-1}$ based on the 20 lowest singlet energies from TD-DFT/B3LYP/6-31G(d) calculations. The effects of various calculation methods and basis sets on the molecular geometry prediction were investigated and compared with 
the experimental single crystal structure results of $2,2^{\prime}$-bipyridine (NP-NP). ${ }^{2}$ Atomic dipole moment corrected Hirshfeld population $(\mathrm{ADCH})$ charge analysis, bond order and nonbonding covalent interaction $(\mathrm{NCI})$ analysis were adopted to investigate the intramolecular interactions using Multiwfn version 3.3 software based on the B3LYP/6-31G(d) optimized molecular structures at ground state $\left(\mathrm{S}_{0}\right){ }^{3,}{ }^{4} \mathrm{NCI}$ isosurface plots were performed with color scaling that the dark blue color represents attractive interactions, while dark red color represents repulsive interactions. ${ }^{5-6}$ All the plotted isosurfaces were demonstrated with reduced density gradient (RDG) of 0.5 and $-0.5<$ $\operatorname{sign}\left(\lambda_{2}\right) \rho<0.5$, where $\operatorname{sign}\left(\lambda_{2}\right)$ means the sign of the second largest eigenvalue of Hessian and $\rho$ represents the electron density.

Reorganization Energy. The charge (hole and electron) mobilities of the soft-conjugated molecules are assessed by using the incoherent hopping model, which assumes a charge transport process between two adjacent molecules in $\mathrm{M}^{ \pm}+\mathrm{M} \rightarrow \mathrm{M}+\mathrm{M}^{ \pm}$, where $\mathrm{M}$ is a neutral molecule interacting with the neighboring cationic or anionic molecule $\left(\mathrm{M}^{ \pm}\right)$to transfer the charge between them. Therefore, the hole (h) or electron (e) reorganization energy $\left(\lambda_{\mathrm{h} / \mathrm{e}}\right)$, in principle, can be calculated in eqs 1 and 2 .

$$
\begin{aligned}
\lambda_{\mathrm{h}} & =\lambda_{+}+\lambda_{1}=\left[E^{+}(M)-E^{+}\left(M^{+}\right)\right]+\left[E\left(M^{+}\right)-E(M)\right] \\
& =\left[E^{+}(M)-E(M)\right]-\left[E^{+}\left(M^{+}\right)-E\left(M^{+}\right)\right]=\mathrm{IP}_{\mathrm{v}}-\mathrm{HEP} \\
\lambda_{e} & =\lambda_{-}+\lambda_{2}=\left[E^{-}(M)-E^{-}\left(M^{-}\right)\right]+\left[E\left(M^{-}\right)-E(M)\right] \\
& =\left[E\left(M^{-}\right)-E^{-}\left(M^{-}\right)\right]-\left[E(M)-E^{-}(M)\right]=\mathrm{EEP}-\mathrm{EA}_{\mathrm{v}}
\end{aligned}
$$


where $\lambda_{+}$is the relaxation energy of a neutral molecule (M) that captured a hole going toward the optimum geometry on the potential energy surface of a cation $\left(\mathrm{M}^{+}\right) ; \lambda_{1}$ is the relaxation energy from a cation $\left(\mathrm{M}^{+}\right)$extracting a hole going toward the $\mathrm{M}$ optimum geometry on the potential energy surface of $\mathrm{M} ; \mathrm{E}^{+}(\mathrm{M})$ and $\mathrm{E}^{+}\left(\mathrm{M}^{+}\right)$are the total energies of the cation species under the optimum geometry of $\mathrm{M}$ and $\mathrm{M}^{+}$, respectively; $\mathrm{E}\left(\mathrm{M}^{+}\right)$and E(M) represent the total energies of the neutral molecule under the optimum geometry of $\mathrm{M}^{+}$and $\mathrm{M}$, respectively; $\mathrm{IP}_{\mathrm{v}}$ and $\mathrm{EA}_{\mathrm{v}}$ are the vertical ionization potential and electron affinity of the molecule, respectively; HEP and EEP are the hole and electron extraction potentials, respectively. The sum of $\lambda_{+}$and $\lambda_{1}$ is the hole reorganization energy $\lambda_{\mathrm{h}}$. Similarly, in the electron transport process, the sum of $\lambda_{-}$and $\lambda_{2}$ is the hole reorganization energy $\left(\lambda_{\mathrm{e}}=\lambda_{-}+\lambda_{2}\right)$, where $\lambda_{-}$is the relaxation energy of a neutral molecule (M) that captured an electron going toward the optimum geometry on the potential energy surface of an anion $\left(\mathrm{M}^{-}\right)$and $\lambda_{2}$ is the relaxation energy from an anion( $\left(\mathrm{M}^{-}\right)$extracting an electron going toward the M optimum geometry on the potential energy surface of $\mathrm{M}^{7}$ 
Table S1. Calculated angle between adjacent aromatic rings $\left(\theta\right.$, in $\left.{ }^{\circ}\right)$ at the ground state using various methods under different basis sets in comparison with the experimental (Exp.) single crystal structure of 2,2'-bipyridine (NP-NP).

\begin{tabular}{|l|c|c|c|c|c|}
\hline NP-NP & HF & B3LYP & CAM-B3LYP & LC-BLYP & $\omega B 97 X D$ \\
\hline 3-21G & 0.0085 & 0.0021 & 0.0014 & 0.0055 & 0.0157 \\
\hline 6-31G & 0.0163 & 0.0107 & 0.0061 & 0.0010 & 0.0019 \\
\hline 6-31G(d) & 0.0208 & 0.0299 & 0.0072 & 0.0089 & 0.0183 \\
\hline 6-311G & 0.0138 & 0.0073 & 0.0127 & 0.0087 & 0.0298 \\
\hline $6-311 G(d)$ & 0.0319 & 0.0133 & 0.0142 & 0.0128 & 0.0212 \\
\hline $6-31 G(d, p)$ & 0.0214 & 0.0006 & 0.0280 & 0.0048 & 0.0281 \\
\hline $6-31+G(d)$ & 0.0045 & 0.0057 & 0.0051 & 0.0031 & 0.0049 \\
\hline $6-31++G(d)$ & 0.0045 & 0.0055 & 0.0049 & 0.0028 & 0.0048 \\
\hline $6-311++G$ & 0.0016 & 0.0009 & 0.0007 & 0.0017 & 0.0006 \\
\hline $6-311++G(d)$ & 0.0047 & 0.0051 & 0.0050 & 0.0030 & 0.0054 \\
\hline Exp. & 0.3083 & 0.3083 & 0.3083 & 0.3083 & 0.3083 \\
\hline
\end{tabular}




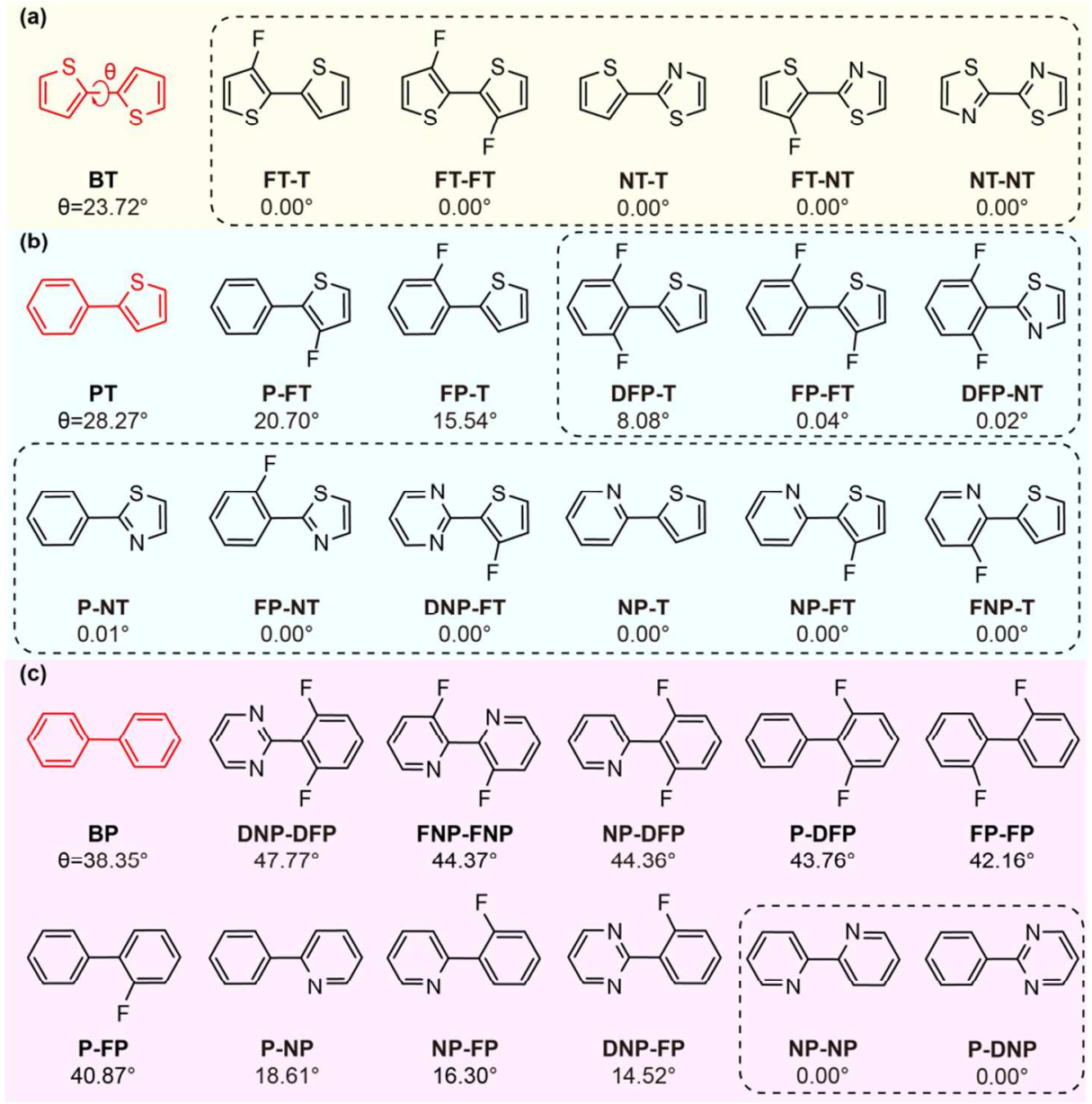

Figure S1. Design of soft-conjugated molecules (SCMs) using six pairs of nonbonding interactions of $\mathrm{F} \cdots \mathrm{S}, \mathrm{F} \cdots \mathrm{N}, \mathrm{F} \cdots \mathrm{H}, \mathrm{S} \cdots \mathrm{N}, \mathrm{S} \cdots \mathrm{H}$, and $\mathrm{N} \cdots \mathrm{H}$ in three kinds of molecular architectures of 5-5 (a), 5-6 (b) and 6-6 (c) pre-bonded aromatic rings. 
(a)
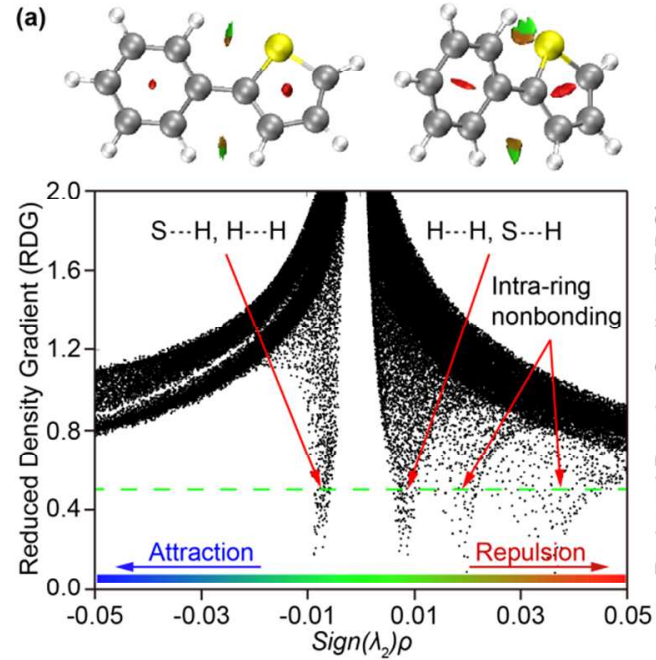

(c)
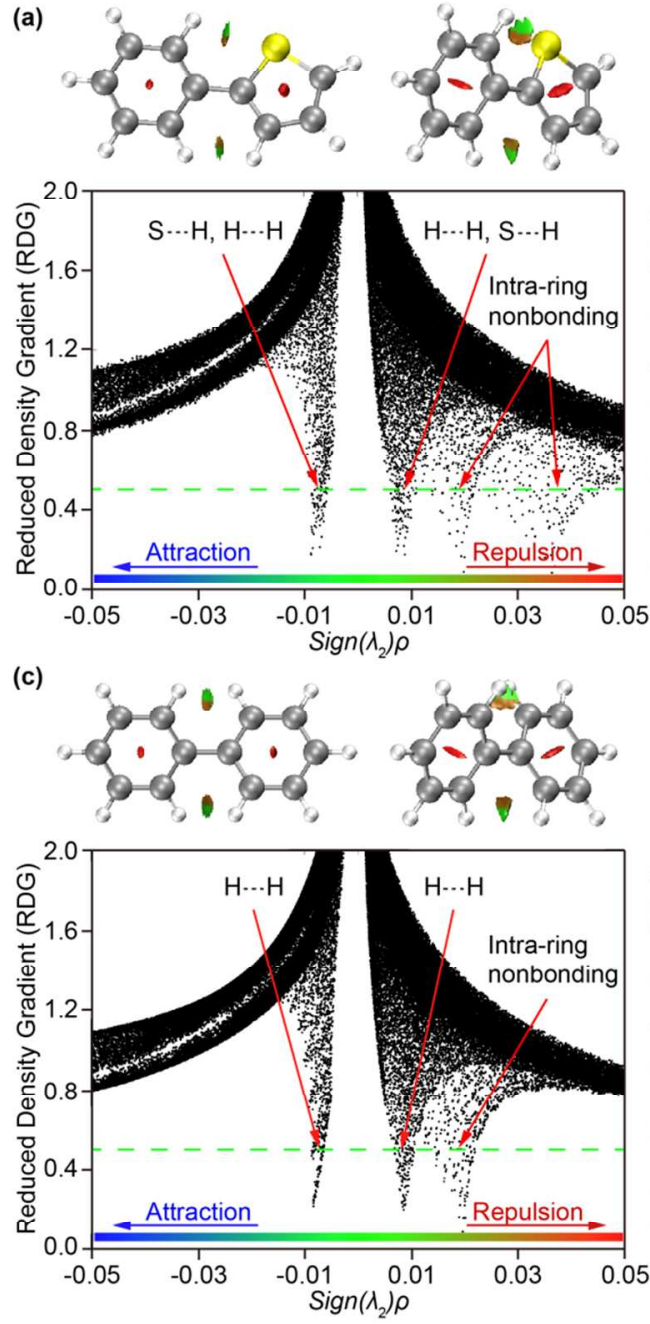

(b)
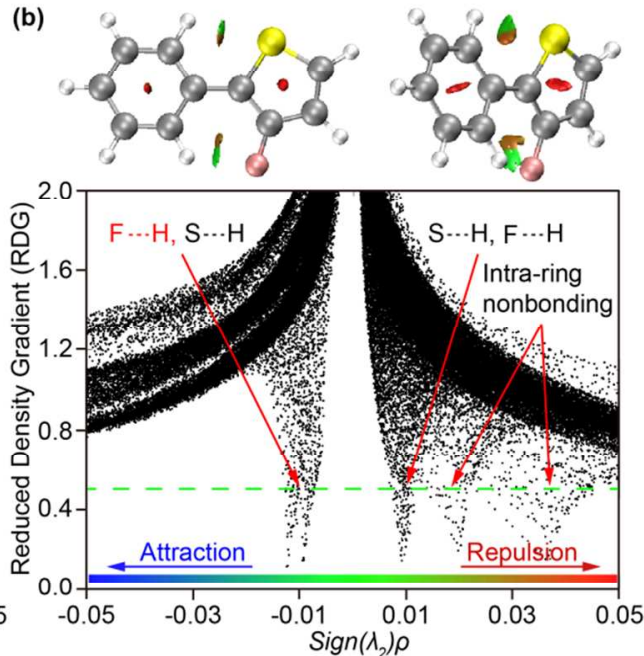

(d)
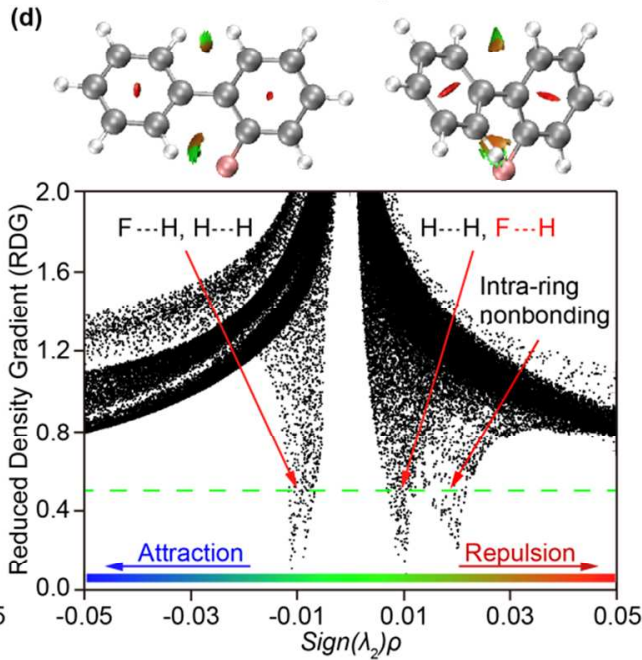

Figure S2. Plots of reduced density gradient (RDG) versus the electron density $(\rho)$ multiplied by the sign of the second Hessian eigenvalue $\left(\lambda_{2}\right)$ with front (left) and side (right) view of low-gradient (RDG $<0.5$ a.u.) RDG maps of PT (a), P-FT (b), BP (c) and P-FP (d). Isovalue of RDG is set to 0.5 , while that of $\operatorname{sign}\left(\lambda_{2}\right) \rho$ on the surface is represented by filling color according to color bar at the bottom from blue to red (from -0.05 to 0.05 a.u.). 
(a)
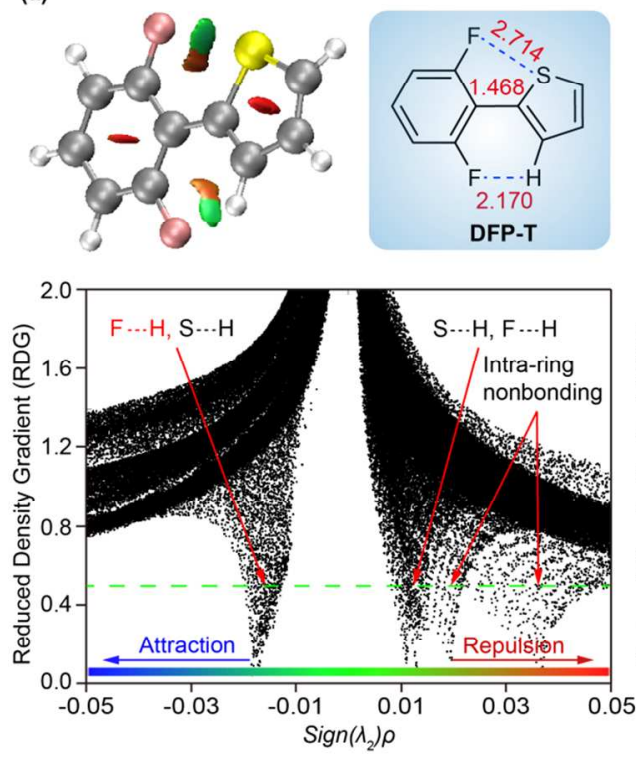
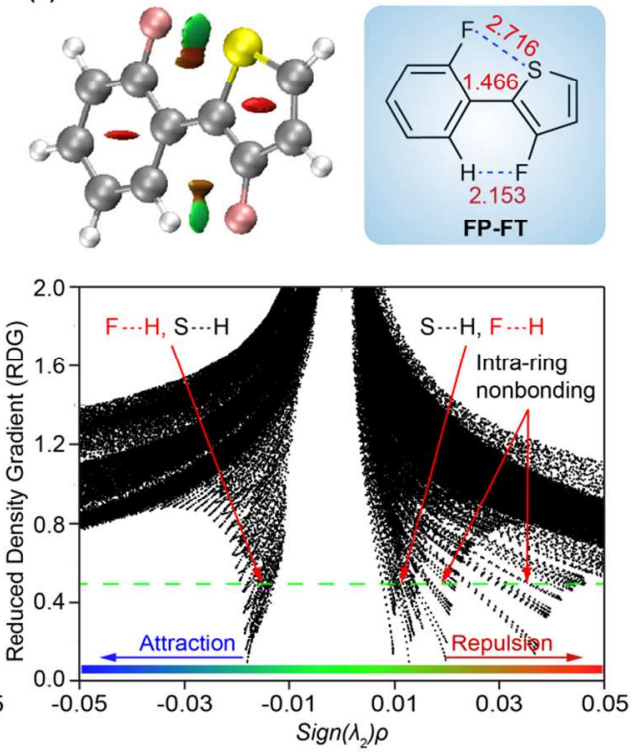

Figure S3. Plots of reduced density gradient (RDG) versus the electron density $(\rho)$ multiplied by the sign of the second Hessian eigenvalue $\left(\lambda_{2}\right)$ with low-gradient $(\mathrm{RDG}<$ 0.5 a.u.) RDG maps (left) and molecules structures (right) of DFP-T (a) and FP-FT (b). Isovalue of RDG is set to 0.5 , while that of $\operatorname{sign}\left(\lambda_{2}\right) \rho$ on the surface is represented by filling color according to color bar at the bottom from blue to red (from -0.05 to 0.05 a.u.). 
(a)
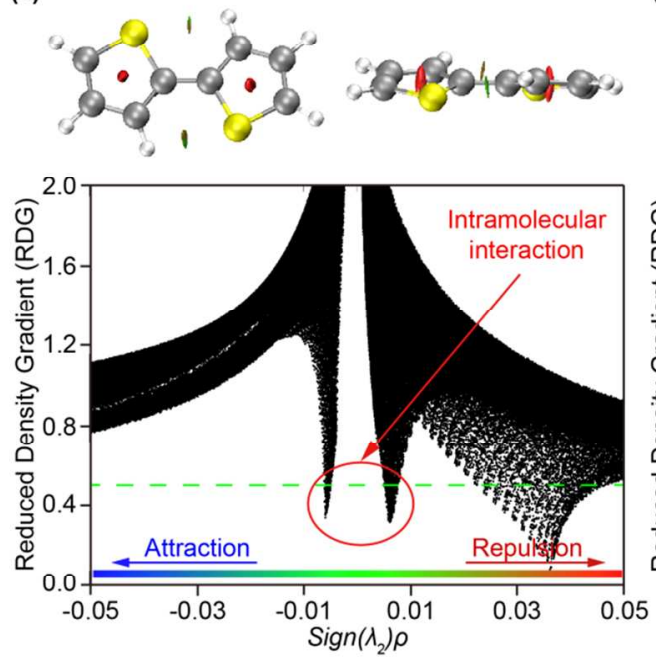

(c)
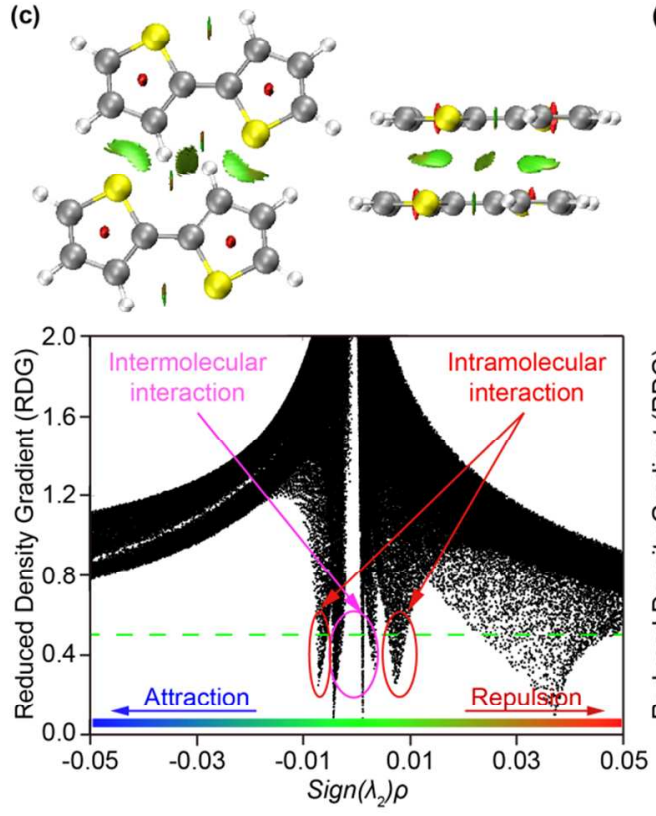

(d)
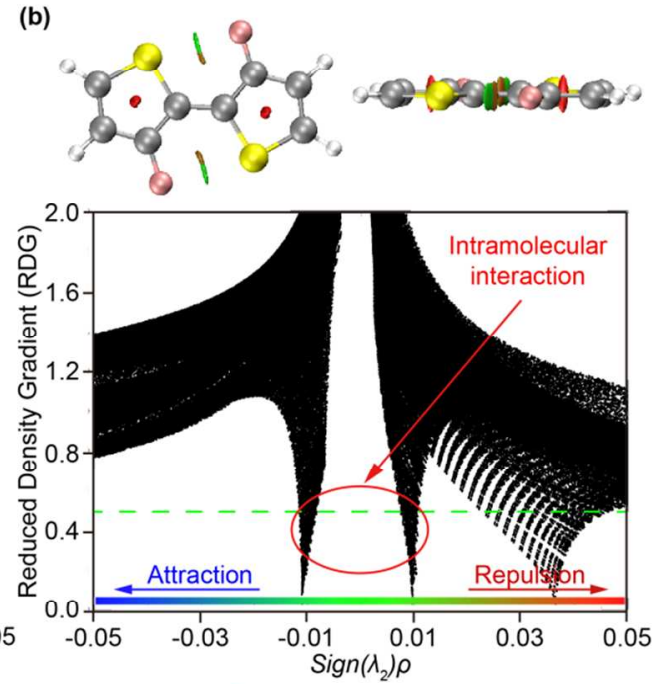

(d)
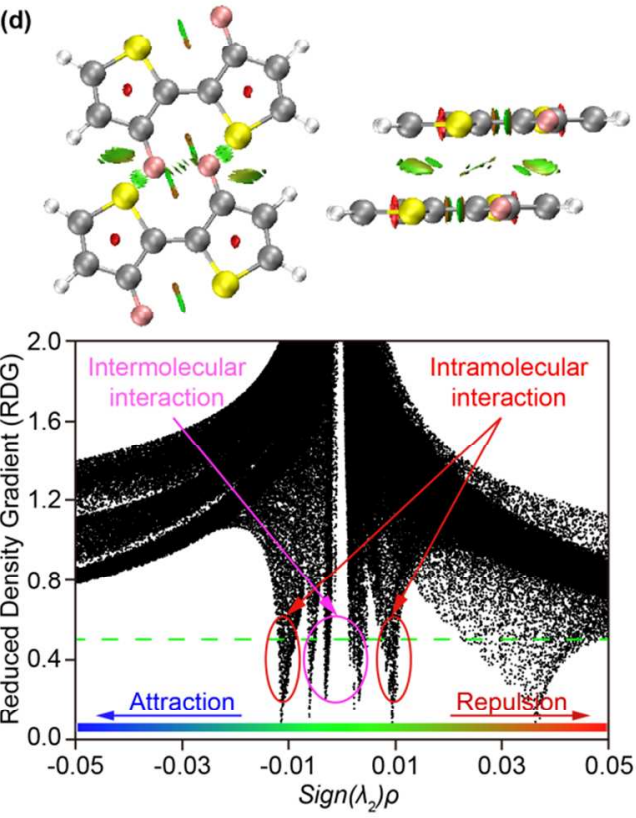

Figure S4. Plots of reduced density gradient (RDG) versus the electron density ( $\rho$ ) multiplied by the sign of the second Hessian eigenvalue $\left(\lambda_{2}\right)$ with front (left) and top (right) view of low-gradient (RDG $<0.5$ a.u.) RDG maps of BT (a), FT-FT (b), dimer BT (c) and dimer FT-FT (d). Isovalue of RDG is set to 0.5 , while that of $\operatorname{sign}\left(\lambda_{2}\right) \rho$ on the surface is represented by filling color according to color bar at the bottom from blue to red (from -0.05 to 0.05 a.u.). 
Table S2. The bonding information of $F \cdots S, F \cdots N, F \cdots H, S \cdots N, S \cdots H$, and $N \cdots H$ nonbonding interactions in SCMs.

\begin{tabular}{|c|c|c|c|c|c|}
\hline Molecule & $\begin{array}{l}\text { Nonbonding } \\
\text { interaction }\end{array}$ & $\begin{array}{l}\text { Distance } \\
(\AA \AA)\end{array}$ & $\begin{array}{c}\text { van der Waals radium } \\
(\AA \hat{)})\end{array}$ & $\Delta \mathrm{D}^{\mathrm{a}}(\AA)$ & $\begin{array}{l}\text { Mulliken } \\
\text { bond order }\end{array}$ \\
\hline \multirow[t]{2}{*}{ FT-T } & $\mathrm{H} \cdots \mathrm{S}$ & 2.951 & $1.10+1.85=2.95$ & 0.001 & 0.012 \\
\hline & $\mathrm{F} \cdots \mathrm{S}$ & 2.946 & $1.35+1.85=3.20$ & -0.254 & 0.036 \\
\hline FT-FT & $\mathrm{F} \cdots \mathrm{S}$ & 2.942 & $1.35+1.85=3.20$ & -0.258 & 0.037 \\
\hline \multirow[t]{2}{*}{ FT-NT } & $\mathrm{F} \cdots \mathrm{S}$ & 2.990 & $1.35+1.85=3.20$ & -0.210 & 0.033 \\
\hline & $\mathrm{N} \cdots \mathrm{S}$ & 3.088 & $1.50+1.85=3.35$ & -0.262 & 0.039 \\
\hline \multirow[t]{2}{*}{ DFP-T } & $\mathrm{F} \cdots \mathrm{S}$ & 2.714 & $1.35+1.85=3.20$ & -0.486 & 0.040 \\
\hline & $\mathrm{H} \cdots \mathrm{F}$ & 2.170 & $1.10+1.35=2.45$ & -0.280 & 0.030 \\
\hline \multirow[t]{2}{*}{ FP-FT } & $\mathrm{F} \cdots \mathrm{S}$ & 2.716 & $1.35+1.85=3.20$ & -0.484 & 0.044 \\
\hline & $\mathrm{H} \cdots \mathrm{F}$ & 2.153 & $1.10+1.35=2.45$ & -0.297 & 0.046 \\
\hline \multirow[t]{2}{*}{ DFP-NT } & $\mathrm{F} \cdots \mathrm{S}$ & 2.706 & $1.35+1.85=3.20$ & -0.494 & 0.040 \\
\hline & $\mathrm{F} \cdots \mathrm{N}$ & 2.675 & $1.35+1.50=2.85$ & -0.175 & -0.003 \\
\hline \multirow[t]{2}{*}{ DNP-FT } & $\mathrm{N} \cdots \mathrm{S}$ & 2.943 & $1.50+1.85=3.35$ & -0.407 & 0.050 \\
\hline & $\mathrm{F} \cdots \mathrm{N}$ & 2.874 & $1.35+1.50=2.85$ & 0.024 & 0.001 \\
\hline \multirow[t]{2}{*}{ FNP-T } & $\mathrm{N} \cdots \mathrm{S}$ & 2.915 & $1.50+1.85=3.35$ & -0.435 & 0.050 \\
\hline & $\mathrm{H} \cdots \mathrm{F}$ & 2.288 & $1.10+1.35=2.45$ & -0.162 & 0.026 \\
\hline P-DNP & $\mathrm{H} \cdots \mathrm{N}$ & 2.453 & $1.10+1.50=2.60$ & -0.162 & 0.040 \\
\hline NP-NP & $\mathrm{H} \cdots \mathrm{N}$ & 2.459 & $1.10+1.50=2.60$ & -0.139 & 0.042 \\
\hline P-TNP & $\mathrm{H} \cdots \mathrm{N}$ & 2.461 & $1.10+1.50=2.60$ & -0.141 & 0.038 \\
\hline
\end{tabular}

a $: \Delta \mathrm{D}=$ Distance - van der Waals radium. 
Table S3. B3LYP/6-31G(d) calculated molecular total energy (E), HOMO and LUMO energy levels, energy band-gap $\left(E_{\mathrm{g}}\right)$ and their variations $(\Delta E, \Delta \mathrm{HOMO}, \Delta \mathrm{LUMO}$, and $\Delta E_{\mathrm{g}}$, respectively) at different $\theta$ of SCMs.

\begin{tabular}{|c|c|c|c|c|c|c|c|c|c|}
\hline Molecule & $\begin{array}{l}\theta \\
\left(^{\circ}\right)\end{array}$ & $\begin{array}{c}E \\
\text { (Hartrees) }\end{array}$ & $\begin{array}{c}\text { HOMO } \\
(\mathrm{eV})\end{array}$ & $\begin{array}{c}\text { LUMO } \\
(\mathrm{eV})\end{array}$ & $\begin{array}{c}E_{\mathrm{g}} \\
(\mathrm{eV})\end{array}$ & $\begin{array}{c}\Delta E \\
\text { (Hartrees) }\end{array}$ & $\begin{array}{c}\Delta \text { HOMO } \\
(\mathrm{eV})\end{array}$ & $\begin{array}{c}\triangle \text { LUMO } \\
(\mathrm{eV})\end{array}$ & $\begin{array}{l}\Delta E_{\mathrm{g}} \\
(\mathrm{eV})\end{array}$ \\
\hline \multirow[t]{3}{*}{ FT-T } & 0.0 & -1204.0451 & -5.530 & -1.279 & 4.251 & & & & \\
\hline & 43.5 & -1204.0430 & -5.746 & -1.109 & 4.637 & 0.0021 & -0.217 & 0.170 & 0.387 \\
\hline & 90.0 & -1204.0397 & -6.391 & -0.696 & 5.695 & 0.0054 & -0.861 & 0.583 & 1.444 \\
\hline \multirow[t]{3}{*}{ FT-FT } & 0.0 & -1303.2742 & -5.589 & -1.297 & 4.293 & & & & \\
\hline & 29.5 & -1303.2721 & -5.690 & -1.250 & 4.441 & 0.0021 & -0.101 & 0.047 & 0.148 \\
\hline & 90.0 & -1303.2675 & -6.463 & -0.814 & 5.649 & 0.0067 & -0.873 & 0.483 & 1.356 \\
\hline \multirow[t]{3}{*}{ FT-NT } & 0.0 & -1220.0941 & -5.897 & -1.593 & 4.304 & & & & \\
\hline & 25.0 & -1220.0919 & -5.957 & -1.538 & 4.420 & 0.0022 & -0.061 & 0.055 & 0.116 \\
\hline & 90.0 & -1220.0823 & -6.551 & -1.093 & 5.458 & 0.0118 & -0.654 & 0.500 & 1.154 \\
\hline \multirow[t]{3}{*}{ DFP-T } & 0.0 & -982.5258 & -5.808 & -1.232 & 4.576 & & & & \\
\hline & 44.1 & -982.5236 & -5.983 & -1.106 & 4.877 & 0.0022 & -0.175 & 0.126 & 0.301 \\
\hline & 90.0 & -982.5199 & -6.328 & -0.717 & 5.612 & 0.0059 & -0.520 & 0.515 & 1.035 \\
\hline \multirow[t]{3}{*}{ FP-FT } & 0.0 & -982.5231 & -5.822 & -1.200 & 4.622 & & & & \\
\hline & 37.5 & -982.5211 & -5.954 & -1.105 & 4.849 & 0.0020 & -0.133 & 0.095 & 0.228 \\
\hline & 90.0 & -982.5163 & -6.346 & -0.686 & 5.659 & 0.0068 & -0.524 & 0.514 & 1.038 \\
\hline \multirow[t]{3}{*}{ FNP-T } & 0.0 & -899.3344 & -5.882 & -1.460 & 4.423 & & & & \\
\hline & 25.1 & -899.3322 & -5.929 & -1.398 & 4.531 & 0.0022 & -0.047 & 0.061 & 0.108 \\
\hline & 90.0 & -899.3212 & -6.257 & -1.067 & 5.190 & 0.0132 & -0.375 & 0.392 & 0.767 \\
\hline \multirow[t]{3}{*}{ P-DNP } & 0.0 & -495.3885 & -6.376 & -1.363 & 5.012 & & & & \\
\hline & 25.4 & -495.3867 & -6.405 & -1.285 & 5.119 & 0.0018 & -0.029 & 0.078 & 0.107 \\
\hline & 90.0 & -495.3752 & -6.545 & -1.076 & 5.469 & 0.0133 & -0.170 & 0.287 & 0.457 \\
\hline \multirow[t]{3}{*}{ NP-NP } & 0.0 & -495.3876 & -6.354 & -1.324 & 5.030 & & & & \\
\hline & 25.3 & -495.3857 & -6.414 & -1.243 & 5.171 & 0.0019 & -0.060 & 0.081 & 0.140 \\
\hline & 90.0 & -495.3732 & -6.663 & -0.729 & 5.934 & 0.0144 & -0.309 & 0.595 & 0.904 \\
\hline \multirow[t]{3}{*}{ P-TNP } & 0.0 & -511.4319 & -6.873 & -1.911 & 4.962 & & & & \\
\hline & 25.2 & -511.4299 & -6.884 & -1.843 & 5.042 & 0.0021 & -0.012 & 0.068 & 0.080 \\
\hline & 90.0 & -511.4173 & -6.920 & -1.494 & 5.425 & 0.0146 & -0.047 & 0.416 & 0.463 \\
\hline
\end{tabular}


Table S4. Vertical ionization potential $\left(\mathrm{IP}_{\mathrm{v}}\right)$, vertical electronic affinity $\left(\mathrm{EA}_{\mathrm{v}}\right)$, extraction potentials (HEP for hole, EEP for electron), and reorganization energies ( $\lambda_{\mathrm{h}}$ for hole, $\lambda_{\mathrm{e}}$ for electron) of soft-conjugated, rigid-conjugated, and non-planar molecules (in eV).

\begin{tabular}{lcccccc}
\hline Molecule & $\mathrm{IP}_{\mathrm{v}}$ & $\mathrm{EA}_{\mathrm{v}}$ & $\mathrm{HEP}$ & $\mathrm{EEP}$ & $\lambda_{\mathrm{h}}$ & $\lambda_{\mathrm{e}}$ \\
\hline FT-T & 7.341 & -0.483 & 6.954 & -0.130 & 0.387 & 0.353 \\
FT-FT & 7.404 & -0.462 & 6.992 & -0.098 & 0.412 & 0.364 \\
FT-NT & 7.733 & -0.199 & 7.318 & 0.184 & 0.415 & 0.384 \\
CT & 7.002 & -0.748 & 6.659 & -0.415 & 0.343 & 0.334 \\
MeT-MeT & 7.553 & -1.056 & 6.632 & -0.272 & 0.920 & 0.783 \\
\hline DFP-T & 7.610 & -0.500 & 7.256 & -0.142 & 0.355 & 0.359 \\
FP-FT & 7.607 & -0.530 & 7.248 & -0.164 & 0.359 & 0.366 \\
FNP-T & 7.689 & -0.296 & 7.367 & 0.044 & 0.322 & 0.340 \\
IT & 7.237 & -0.855 & 6.935 & -0.513 & 0.302 & 0.342 \\
MeP-MeT & 7.710 & -1.218 & 6.990 & -0.432 & 0.719 & 0.786 \\
\hline P-DNP & 8.182 & -0.398 & 7.949 & 0.001 & 0.233 & 0.398 \\
NP-NP & 8.132 & -0.426 & 7.880 & -0.012 & 0.252 & 0.414 \\
P-TNP & 8.752 & 0.097 & 8.511 & 0.497 & 0.241 & 0.399 \\
FL & 7.482 & -0.969 & 7.220 & -0.648 & 0.262 & 0.321 \\
P-DMeP & 8.072 & -1.692 & 7.384 & -0.681 & 0.687 & 1.011 \\
\hline
\end{tabular}


Table S5. TD-B3LYP/6-31G(d) predicted absorption peak wavelengths $\left(\lambda_{\mathrm{abs}}\right)$, main transition configurations, and oscillator strengths.

\begin{tabular}{lcclc}
\hline Molecule & Electron transition & $\lambda_{\text {abs }}(\mathrm{nm})$ & Main transition configuration & Oscillator strength \\
\hline FT-T & $\mathrm{S}_{0} \rightarrow \mathrm{S}_{1}$ & 304.60 & HOMO $\rightarrow$ LUMO $(0.99)$ & 0.4322 \\
\hline FT-FT & $\mathrm{S}_{0} \rightarrow \mathrm{S}_{1}$ & 299.54 & HOMO $\rightarrow$ LUMO $(0.99)$ & 0.4462 \\
\hline FT-NT & $\mathrm{S}_{0} \rightarrow \mathrm{S}_{1}$ & 302.68 & HOMO $\rightarrow$ LUMO $(0.98)$ & 0.4166 \\
\hline CT & $\mathrm{S}_{0} \rightarrow \mathrm{S}_{1}$ & 309.50 & HOMO $\rightarrow$ LUMO $(0.99)$ & 0.3518 \\
\hline MeT-MeT & $\mathrm{S}_{0} \rightarrow \mathrm{S}_{1}$ & 266.35 & HOMO $\rightarrow$ LUMO $(0.92)$ & 0.1873 \\
\hline DFP-T & $\mathrm{S}_{0} \rightarrow \mathrm{S}_{1}$ & 282.96 & HOMO $\rightarrow$ LUMO $(0.98)$ & 0.4961 \\
\hline FP-FT & $\mathrm{S}_{0} \rightarrow \mathrm{S}_{1}$ & 280.67 & HOMO $\rightarrow$ LUMO $(0.95)$ & 0.4748 \\
\hline FNP-T & $\mathrm{S}_{0} \rightarrow \mathrm{S}_{1}$ & 300.56 & HOMO $\rightarrow$ LUMO $(0.92)$ & 0.3716 \\
\hline IT & $\mathrm{S}_{0} \rightarrow \mathrm{S}_{1}$ & 284.08 & HOMO $\rightarrow$ LUMO $(0.96)$ & 0.3765 \\
\hline MeP-MeT & $\mathrm{S}_{0} \rightarrow \mathrm{S}_{1}$ & 255.05 & HOMO $\rightarrow$ LUMO $(0.86)$ & 0.1095 \\
\hline P-DNP & $\mathrm{S}_{0} \rightarrow \mathrm{S}_{1}$ & 298.69 & HOMO-2 $\rightarrow$ LUMO $(0.99)$ & 0 \\
& $\mathrm{~S}_{0} \rightarrow \mathrm{S}_{5}$ & 262.66 & HOMO $\rightarrow$ LUMO $(0.91)$ & 0.4617 \\
\hline NP-NP & $\mathrm{S}_{0} \rightarrow \mathrm{S}_{1}$ & 290.42 & HOMO-1 $\rightarrow$ LUMO $(0.98)$ & 0.0019 \\
\hline P-TNP & $\mathrm{S}_{0} \rightarrow \mathrm{S}_{3}$ & 264.78 & HOMO $\rightarrow$ LUMO $(0.92)$ & 0.4518 \\
& $\mathrm{~S}_{0} \rightarrow \mathrm{S}_{1}$ & 301.41 & HOMO-2 $\rightarrow$ LUMO $(0.99)$ & 0 \\
\hline FL & $\mathrm{S}_{0} \rightarrow \mathrm{S}_{6}$ & 265.22 & HOMO $\rightarrow$ LUMO $(0.94)$ & 0.4347 \\
\hline P-DMeP & $\mathrm{S}_{0} \rightarrow \mathrm{S}_{1}$ & 265.17 & HOMO $\rightarrow$ LUMO $(0.41)$ & 0.1754 \\
\hline & $\mathrm{S}_{0} \rightarrow \mathrm{S}_{1}$ & 234.46 & HOMO- $\rightarrow$ LUMO $+3(0.34)$ & 0.0047 \\
\hline & & & & \\
\hline
\end{tabular}


Table S6. TD-B3LYP/6-31G(d) predicted emission peak wavelengths $\left(\lambda_{\mathrm{em}}\right)$, main transition configurations, and oscillator strengths.

\begin{tabular}{lcclc}
\hline Molecule & Electron transition & $\lambda_{\text {em }}(\mathrm{nm})$ & Main transition configuration & Oscillator strength \\
\hline FT-T & $\mathrm{S}_{1} \rightarrow \mathrm{S}_{0}$ & 350.99 & HOMO $\rightarrow$ LUMO $(1.00)$ & 0.4390 \\
\hline FT-FT & $\mathrm{S}_{1} \rightarrow \mathrm{S}_{0}$ & 342.09 & HOMO $\rightarrow$ LUMO $(1.00)$ & 0.4624 \\
\hline FT-NT & $\mathrm{S}_{1} \rightarrow \mathrm{S}_{0}$ & 352.81 & HOMO $\rightarrow$ LUMO $(0.99)$ & 0.4166 \\
\hline CT & $\mathrm{S}_{1} \rightarrow \mathrm{S}_{0}$ & 365.63 & HOMO $\rightarrow$ LUMO $(1.00)$ & 0.3328 \\
\hline DFP-T & $\mathrm{S}_{1} \rightarrow \mathrm{S}_{0}$ & 319.72 & HOMO $\rightarrow$ LUMO $(0.99)$ & 0.5324 \\
FP-FT & $\mathrm{S}_{1} \rightarrow \mathrm{S}_{0}$ & 318.92 & HOMO $\rightarrow$ LUMO $(0.99)$ & 0.5230 \\
FNP-T & $\mathrm{S}_{1} \rightarrow \mathrm{S}_{0}$ & 343.92 & HOMO $\rightarrow$ LUMO $(0.94)$ & 0.3015 \\
\hline IT & $\mathrm{S}_{1} \rightarrow \mathrm{S}_{0}$ & 332.03 & HOMO $\rightarrow$ LUMO $(0.97)$ & 0.3614 \\
\hline P-DNP & $\mathrm{S}_{1} \rightarrow \mathrm{S}_{0}$ & 465.68 & HOMO $\rightarrow$ LUMO $(0.99)$ & 0 \\
& $\mathrm{~S}_{3} \rightarrow \mathrm{S}_{0}$ & 278.68 & HOMO-1 $\rightarrow$ LUMO $(0.99)$ & 0.6931 \\
\hline NP-NP & $\mathrm{S}_{1} \rightarrow \mathrm{S}_{0}$ & 355.32 & HOMO-1 $\rightarrow$ LUMO $(0.99)$ & 0.0010 \\
& $\mathrm{~S}_{3} \rightarrow \mathrm{S}_{0}$ & 281.74 & HOMO $\rightarrow$ LUMO $(0.95)$ & 0.5943 \\
\hline P-TNP & $\mathrm{S}_{1} \rightarrow \mathrm{S}_{0}$ & 493.50 & HOMO $\rightarrow$ LUMO $(0.99)$ & 0 \\
& $\mathrm{~S}_{5} \rightarrow \mathrm{S}_{0}$ & 274.79 & HOMO-1 $\rightarrow$ LUMO $(0.98)$ & 0.6354 \\
\hline FL & $\mathrm{S}_{1} \rightarrow \mathrm{S}_{0}$ & 299.20 & HOMO $\rightarrow$ LUMO $(0.94)$ & 0.4072 \\
\hline
\end{tabular}




\section{References:}

(1) Zheng, C.; An, Z.; Nakai, Y.; Tsuboi, T.; Wang, Y.; Shi, H.; Chen, R.; Li, H.; Ji, Y.; Li, J.; et al.

Relationships between Main-chain Chirality and Photophysical Properties in Chiral Conjugated Polymers.

J. Mater. Chem. C 2014, 2, 7336-7347.

(2) Kuhn, F. E.; Groarke, M.; Bencze, E.; Herdtweck, E.; Prazeres, A.; Santos, A. M.; Calhorda, M. J.;

Romao, C. C.; Goncalves, I. S.; Lopes, A. D.; et al. Octahedral Bipyridine and Bipyrimidine

Dioxomolybdenum(vi) Complexes: Characterization, Application in Catalytic Epoxidation, and Density

Functional Mechanistic Study. Chem. Eur. J. 2002, 8, 2370-2383.

(3) Lu, T.; Chen, F. W. Atomic Dipole Moment Corrected Hirshfeld Population Method. J Theor Comput Chem 2012, 1, 163-183.

(4) Lu, T.; Chen, F. W. Multiwfn: A Multifunctional Wavefunction Analyzer. J. Comput. Chem. 2012, 33 , $580-592$.

(5) Jackson, N. E.; Savoie, B. M.; Kohlstedt, K. L.; de la Cruz, M. O.; Schatz, G. C.; Chen, L. X.; Ratner, M. A. Controlling Conformations of Conjugated Polymers and Small Molecules: The Role of Nonbonding Interactions. J. Am. Chem. Soc 2013, 135, 10475-10483.

(6) Li, Y.; Chen, S.; Ren, F. Theoretical Insights into the Structures and Mechanical Properties of HMX/NQ Cocrystal Explosives and their Complexes, and the Influence of Molecular Ratios on their Bonding Energies. J. Mol. Model. 2015, 21, 245-257.

(7) Yan, M.; Tao, Y.; Chen, R.; Zheng, C.; An, Z.; Huang, W. Computational Design and Selection of Optimal Building Blocks and Linking Topologies for Construction of High-performance Host Materials. RSC Adv. 2012, 2, 7860-7867. 